\title{
Placental histopathology in late preterm infants: clinical implications
}

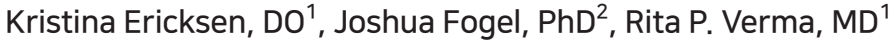 \\ ${ }^{1}$ Department of Pediatrics, Nassau University Medical Center, East Meadow, NY, USA; ${ }^{2}$ Brooklyn College, Brooklyn, NY, USA
}

Background: The etiopathogenesis of late preterm (LPT) birth is undetermined. Placental histopathology, which reflects an adverse intrauterine environment and is reportedly associated with preterm labor and neonatal morbidities, has not been studied in LPT infants.

Purpose: We investigated placental pathological lesion as markers of an adverse intrauterine environment during LPT labor.

Methods: This retrospective case-control study compared placental histopathological and clinical variables between LPT and term neonates. Placental variables included chorioamnionitis, funisitis, hemorrhage, abruption, infarction, calcification, and syncytial knots. Maternal variables included age, substance abuse, pregnancyassociated diabetes mellitus and hypertension, duration of rupture of membrane, antibiotic use, and magnesium sulfate, whereas, those of neonates included gestational age, birth weight, race, sex, and Apgar scores. Standard statistical proedures were applied to analyze the data.

Results: Chorioamnionitis $(50 \%$ vs. $17.8 \%, P<0.001)$ and funisitis ( $20 \%$ vs. $4.4 \%, P=0.002)$ were more common in term infants. Placental infarction rate was insignificantly higher in LPT infants ( $25.6 \%$ vs. $14.3 \%, P=0.08)$. The mothers in the LPT group were older (30.4 years vs. 28.1 years, $P=0.05$; odds ratio [OR], 1.06; 95\% confidence interval [CI], 0.998-1.12, $P=0.056)$ and more often suffered from hypertension (28.9 vs. $12.9 \%, P=0.02$ ), and received magnesium sulfate ( 48.9 vs. $20 \%, P<0.001$; OR, 2.86; 95\% CI, 1.12-7.29, $P<0.05)$. Duration of rupture of membrane was higher in term infants (13.6 hours vs. 9.1 hours, $P<0.001)$. Chorioamnionitis (OR, 0.33; $95 \% \mathrm{CI}, 0.13-0.79 ; P<0.05$ ) was associated with a lower risk of LPT delivery.

Conclusion: Placental infection is not a risk factor for LPT births. There is a nonsignificant predominance of vascular anomalies in LPT placentas. Higher maternal age, magnesium sulfate therapy, and maternal hypertension are clinical risk factors for LPT labor.

Key words: Late preterm infants, Placental histopathology, Vasculopathy, Gestation associated hypertension, Chorioam. nionitis

\section{Key message}

Question: Placental histopathology and its clinical implications in late preterm infants.

Finding: Placental vascular anomalies are more, and placental inflammation less common in late preterm infants compared to term. Higher maternal age, magnesium sulfate therapy and hypertension are clinical risk factors associated with late preterm delivery.

Meaning: Prevention and aggressive management of hypertension, and conception before 30 years of age might be effective in preventing late preterm births.

\section{Introduction}

Late preterm (LPT) infants, born at 33, 0/7 to 36, 6/7 weeks of gestational age (GA) constitute $71 \%$ of all preterm births and $33 \%$ of all neonatal intensive care unit (NICU) admissions. ${ }^{1-3)}$ Their birth distribution has increased by over 25\% since 1990 . LPT neonates are at increased risks for mortality, transient tachy. pnea of newborn, respiratory distress syndrome, hypoglycemia, intraventricular-periventricular hemorrhage (IVH), adverse neurodevelopment and attention deficit disorders compared to term (T) infants. ${ }^{2-5)}$ They have higher rates for admission to NICU and postdischarge hospitalization. Their length of hospital stay, resource utilization and total cost of care are significantly higher and in later life they suffer from poorer social adjustment and financial productivity.

The exact etiopathogenesis of LPT labor is undetermined. Ninety-three percent of all LPT births is spontaneous or associated with spontaneous rupture of membrane. ${ }^{2,3)}$ Prophylactic cervical cerclage, bed rest, tocolysis, or home monitoring have been found to be unsuccessful in preventing LPT birth. Understanding the etiopathogenesis and avoiding potential risk factors might decrease the incidence of LPT births, their related morbidities, and cost of care.

Placental lesions reflect the intrauterine environment and have been associated with preterm births and adverse outcomes in neonates. ${ }^{6-8)}$ Chorioamnionitis $(\mathrm{CHO})$ and funisitis $(\mathrm{FN})$ are

Corresponding author: Rita P. Verma, MD. Department of Pediatrics, Nassau University Medical Center, 2201 Hempstead Turnpike, East Meadow, NY 11554, USA 凶E-mail: ritaverma@aol.com, https://orcid.org/0000-0002-6622-124X

Received: 11 January, 2019, Revised: 2 August, 2019, Accepted: 13 August, 2019

This is an open-access article distributed under the terms of the Creative Commons Attribution Non-Commercial License (http://creativecommons.org/licenses/bync/4.0/) which permits unrestricted non-commercial use, distribution, and reproduction in any medium, provided the original work is properly cited.

Copyright (c) 2020 by The Korean Pediatric Society 
documented to be associated with premature rupture of membranes (ROMs), IVH, retinopathy of prematurity and delivery of very low birth weight infants, whereas, placental vascular obstructive lesions are correlated with necrotizing enterocolitis. The literature on placental histopathology $(\mathrm{PH})$ in neonates is comprised almost entirely of reports in the prematurely born infants at $<32$ weeks of GA. There is scant information on PH in the LPT infants and none of the referred reports has described the pathological findings exclusively in this population.

In this study, we evaluated the basic PH in infants born during LPT gestational weeks, along with relevant maternal - neonatal clinical characteristics. The objective was to identify specific placental histopathological findings, that could serve as markers of adverse intrauterine environment during LPT labor, which resulted in the delivery of an LPT infant.

\section{Methods}

This was a retrospective case-control study conducted at a suburban academic hospital in New York over a 15-month study period during 2014 and 2015. The project was approved by the Institutional Review Board prior to commencement (IRB No. 15-041).

\section{Population}

All inborn infants admitted consecutively to the NICU during the specified study period with GAs between 33, 0/7 and 40,0/7 weeks were enrolled in the study. The clinical and histopathological data were retrieved from the computer files by reviewing progress notes and laboratory, as well as micro pathological reports.

\section{Variables}

The $\mathrm{PH}$ was evaluated for the presence of $\mathrm{CHO}, \mathrm{FN}$, hemorrhage, abruption, infarction, calcification, and syncytial knots.

The maternal variables studied were as follows: age; parity; infection status, including chlamydia, rubella, syphilis, human immunodeficiency virus and rectovaginal group B streptococcal colonization; the presence of pregnancy-associated diabetes mellitus and pregnancy-associated hypertension (PAH); duration of ROM in hours; substance abuse, including cocaine, cannabinoids, opiates, nicotine, and others; receipt of antibiotics within 24 hours of delivery and antenatal receipt of magnesium sulfate $\left(\mathrm{MgSO}_{4}\right)$. The following neonatal variables were noted: GA, birth weight, race, sex, and Apgar scores.

\section{Statistical analysis}

The data were analyzed under 2 groups: LPT, which comprised of infants born with GA of 33,0/7 and 36, 6/7 weeks, and $\mathrm{T}$, which included term infants who were born between 37 , $0 / 7$ and 40,0/7 weeks of gestation. Variables were compared via Student $t$ test, chi-square and Fisher exact tests for univariate analyses, whereas, a multivariate analysis was performed by utilizing linear regression and multiple logistic regressions for continuous and categorical outcome variables respectively. Variables with skewed distribution, such as, Apgar score at 5 minutes of life and duration of the ROM were logarithmic transformed. ROM had the value 1 added to each individual number and then logarithmic transformed, as value of 0 precluded the logarithmic transformation. All $P$ values were 2 tailed and a $P$ value of $<0.05$ was considered significant. IBM SPSS Statistics ver. 23.0 (IBM Co., Armonk, NY, USA) was used for analyses.

\section{Results}

A total of 160 newborn infants were included in the study, with 90 in T and 70 in LPT group. According to the univariate analyses, $\mathrm{CHO}(50 \%$ vs. $17.8 \%, P<0.001)$ and $\mathrm{FN}(20 \%$ vs. $4.4 \%, P=0.002)$ were more common in $\mathrm{T}$ infants (Table1). The occurrence of placental infarction, although higher in LPT infants $(25.6 \%$ vs. $14.3 \%$ in $\mathrm{T}, P=0.08)$ ) did not reach significance. The rest of the placental histopathological variables did not differ significantly between the groups. The mothers in LPT group were older (30.4 years vs. 28.1 years, $P=0.05$ ), suffered from PAH more often (28.9\% vs. $12.9 \%, P=0.02)$ and a higher percentage of them received $\mathrm{MgSO} 4$ antenatally (48.9\% vs. $20 \%, P<0.001)$ than T. The duration of ROM was higher in $\mathrm{T}$ infants (13.6 hours vs. 9.1 hours in LPT, $P<0.001)$. In the multivariate logistic regression analysis, which included the significant variables identified via univariate analysis, $\mathrm{CHO}(\mathrm{OR}$, 0.33 ; 95\% CI, 0.13-0.79; $P<0.05)$ was associated with lower and receipt of $\mathrm{MgSO} 4$ (OR, 2.86; 95\% CI, 1.12-7.29; $P<0.05$ ) with higher odds of LPT delivery (Table 2). Maternal age showed a significant trend (OR, 1.06; 95\% CI, 0.998-1.12; $P=0.056$ ) towards being higher in LPT neonates.

\section{Discussion}

PH has been studied extensively. However, only a few reports have analyzed it in LPT neonates, ${ }^{6-8)}$ and none of those included LPT infants exclusively in their study. Stanek ${ }^{9}$ found clinical conditions linked to in-utero hypoxia, such as preeclampsia and diabetes mellitus, and their placental associations, such as atherosis, membrane chorionic microcysts, chorangiosis, intervillous thrombi to be associated with mid third trimester deliveries. In a recent report by Catov et al., ${ }^{10)}$ occurrences of placental malperfusion, vasculopathy, infarction and syncytial knots were found to be higher, while those of $\mathrm{CHO}$ and intrauterine inflammation lower in LPT infants compared to term infants. Our results regarding intrauterine infection are in accordance with their findings and exclude infection as the potential precipitating event in LPT labor. There is a growing awareness of sterile inflammation as a causative factor in LPT deliveries. ${ }^{11)}$ The occurrences of abruption, infarction, and syncytial knots, although higher in LPT infants compared to term did not reach 
Table 1. Results of univariate analysis of neonatal, maternal, and placental variables

\begin{tabular}{|c|c|c|c|}
\hline Variable & Full term $(n=70)$ & Late preterm $(n=90)$ & $P$ value \\
\hline \multicolumn{4}{|l|}{ Placental histopathology } \\
\hline Chorioamnionitis & $35(50.0)$ & $16(17.8)$ & $<0.001$ \\
\hline Funisitis & $14(20.0)$ & $4(4.4)$ & 0.002 \\
\hline Hemorrhage & $7(10.0)$ & $4(4.4)$ & 0.21 \\
\hline Placental Abruption & $1(1.4)$ & $4(4.4)$ & 0.39 \\
\hline Infarction & $10(14.3)$ & $23(25.6)$ & 0.08 \\
\hline Syncytial knots & $8(11.4)$ & $19(21.1)$ & 0.11 \\
\hline Calcifications & $23(32.9)$ & $18(20.0)$ & 0.07 \\
\hline \multicolumn{4}{|l|}{ Infant variables } \\
\hline Birth weight (g) & $3,208.8 \pm 530.11$ & $2,024.4 \pm 518.98$ & $<0.001$ \\
\hline Gestational age (wk) & $38.9 \pm 1.38$ & $33.0 \pm 1.98$ & $<0.001$ \\
\hline Male sex & $36(51.4)$ & $55(61.1)$ & 0.22 \\
\hline \multicolumn{4}{|l|}{ Race/ethnicity } \\
\hline White & $11(15.7)$ & $11(12.2)$ & \\
\hline Black & $18(25.7)$ & $24(26.7)$ & \\
\hline Hispanic & $36(51.4)$ & $47(52.2)$ & \\
\hline Other & $5(7.1)$ & $8(8.9)$ & \\
\hline Growth & & & 0.99 \\
\hline Appropriate for gestational age & $59(84.3)$ & $76(84.4)$ & \\
\hline Large for gestational age & $5(7.1)$ & $6(6.7)$ & \\
\hline Small for gestational age & $6(8.6)$ & $8(8.9)$ & \\
\hline Apgar at $1 \mathrm{~min}$ & $7.3 \pm 2.45$ & $7.4 \pm 1.89$ & 0.89 \\
\hline Apgar at $5 \mathrm{~min}$ & $8.2 \pm 1.55$ & $8.2 \pm 1.10$ & 0.66 \\
\hline \multicolumn{4}{|l|}{ Maternal variable } \\
\hline Age (yr) & $28.1 \pm 5.99$ & $30.4 \pm 6.49$ & 0.02 \\
\hline Substance abuse & $2(2.9)$ & $3(3.3)$ & 1.00 \\
\hline Gestational diabetes mellitus & $5(7.1)$ & $7(7.8)$ & 0.88 \\
\hline Maternal hypertension & 9 (12.9) & $26(28.9)$ & 0.02 \\
\hline Magnesium sulfate (maternal treatment) & $14(20.0)$ & $44(48.9)$ & $<0.001$ \\
\hline Antibiotics (maternal treatment) & $42(60.0)$ & $53(58.9)$ & 0.89 \\
\hline Duration of rupture of membrane (hr) & $13.6 \pm 13.54$ & $9.1 \pm 15.66$ & $<0.001$ \\
\hline
\end{tabular}

Values are presented as number (\%) or mean \pm standard deviation.

Boldface indicates a statistically significant difference with $P<0.05$.

Table 2. Results of multivariate analyses for late preterm infants

\begin{tabular}{lcc}
\hline Variable & \multicolumn{1}{c}{ Univariate } & Multivariate \\
\hline $\begin{array}{l}\text { Placental histopathology } \\
\text { Chorioamnionitis }\end{array}$ & $0.22(0.11-0.44)^{* * *}$ & $0.33(0.13-0.79)^{*}$ \\
Funisitis & $0.19(0.06-0.59)^{* *}$ & $0.59(0.15-2.37)$ \\
Maternal variable & & \\
Age (yr) & $1.06(1.01-1.12)^{*}$ & $1.06(0.998-1.12)$ \\
Maternal hypertension & $2.75(1.19-6.35)^{*}$ & $1.09(0.36-3.26)$ \\
Receipt of Magnesium sulfate & $3.83(1.87-7.84)^{* * *}$ & $2.86(1.12-7.29)^{*}$ \\
Rupture of membrane (hr) & $0.34(0.20-0.59)^{* * *}$ & $1.002(0.98-1.03)$ \\
\hline $\begin{array}{l}\text { Values are presented as odds ratio (confidence interval). } \\
{ }^{*} P<0.05,{ }^{* *} P<0.01,{ }^{* * *} P<0.001\end{array}$ &
\end{tabular}

significance, most likely due to a beta error, which is a limitation of this study. The higher occurrences of placental vascular lesions apparently were consequent to the associated maternal hypertension. The findings that the placental inflammatory lesions are less and vascular lesions more marked in LPT neonates are in contrast to those in infants who are born before 32 weeks or at extremely low GA and exhibit $\mathrm{CHO}$ and $\mathrm{FN}$ as the predominant lesions in their placenta. ${ }^{6-8,12)}$

In regards to clinical variables, we found gestational hypertension, antenatal use of $\mathrm{MgSO}_{4}$, and increased maternal age to be significant risk factors for LPT deliveries. These results are consistent with past reports on this subject. Haroon et al..$^{5)}$ documented higher maternal age, maternal diabetes mellitus, hy. pertension, urinary tract infection and prolonged ROM (PROM) as risk factors for LPT compared to term deliveries. In a systematic review, Machado Júnior et al. ${ }^{11)}$ noted that, while, the etiology in most cases of LPT births is unknown, mothers with Factor $\mathrm{V}$ Leiden carrier status, age $<20$ years and those with no antenatal care are at increased risk. None of the studies in their review described PH solely in the specific LPT population. Laughon et al. ${ }^{13)}$ reported that $31 \%$ of all LPT infants are delivered via medical interruption of pregnancy, while $36 \%$ are consequent to spontaneous labor due to unknown etiology and the rest $32 \%$ are associated with PROM. They advocated reviewing pregnancy termination routines and increasing the use of tocolytics and 
steroids as strategies to address the issue.

The placental histopathological findings, examined exclusively in the LPT neonates in this study, provide significant insight into the adverse intrauterine conditions and timing of preterm labor. In association with a shorter duration of ROM, placental inflammation is less common and placental vascular lesions more pronounced in LPT infants, whereas, intrauterine infection is the major etiopathogenic factor in the onset of premature labor before 32 weeks of gestation. ${ }^{6-8,12)}$ These findings suggest that while controlling maternal infection may result in a decrease in the birth of extremely low GA infants, prevention and aggressive management of gestational hypertension might be efficacious in extending the duration of pregnancy and decreasing the birth rate and distribution of LPT infants. Formulating strategies for decreasing the occurrence of maternal hypertension and counselling in favor of lower maternal age might prove to be effective measures in the prevention of birth of LPT infants and decreasing their NICU admission rate and overall cost of care.

\section{Conflicts of interest}

No potential conflict of interest relevant to this article was reported.

\section{References}

1. Shapiro-Mendoza CK, Lackritz EM. Epidemiology of late and moderate preterm birth. Semin Fetal Neonatal Med 2012;17:120-5.

2. Loftin RW, Habli M, Snyder CC, Cormier CM, Lewis DF, Defranco EA. Late preterm birth. Rev Obstet Gynecol 2010;3:10-9.

3. Kugelman A, Colin AA. Late preterm infants: near term but still in a critical developmental time period. Pediatrics 2013;132:741-51.

4. Darcy AE. Complications of the late preterm infant. J Perinat Neonatal Nurs 2009;23:78-86.

5. Haroon A, Ali SR, Ahmed S, Maheen H. Short-term neonatal outcome in late preterm vs. term infants. J Coll Physicians Surg Pak 2014;24:34-8.

6. Roescher AM, Timmer A, Erwich JJ, Bos AF. Placental pathology, perinatal death, neonatal outcome, and neurological development: a systematic review. PLoS One 2014;9:e89419.

7. Perrone S, Toti P, Toti MS, Badii S, Becucci E, Gatti MG, et al. Perinatal outcome and placental histological characteristics: a single-center study. J Matern Fetal Neonatal Med 2012;25 Suppl 1:110-3.

8. Moscuzza F, Belcari F, Nardini V, Bartoli A, Domenici C, Cuttano A, et al. Correlation between placental histopathology and fetal/neonatal outcome: chorioamnionitis and funisitis are associated to intraventricular haemorrage and retinopathy of prematurity in preterm newborns. Gynecol Endocrinol 2011;27:319-23.

9. Stanek J. Comparison of placental pathology in preterm, late-preterm, near-term, and term births. Am J Obstet Gynecol 2014;210:234.e1-6.

10. Catov JM, Scifres CM, Caritis SN, Bertolet M, Larkin J, Parks WT. Neonatal outcomes following preterm birth classified according to placental features. Am J Obstet Gynecol 2017;216:411.e1-411.e14.

11. Machado Júnior LC, Passini Júnior R, Rodrigues Machado Rosa I. Late prematurity: a systematic review. J Pediatr (Rio J) 2014;90:221-31.

12. Verma RP, Zhao Y, Niwas R, Kaplan C. Isolated placental inflammation and vasculopathy: clinical implications in the extremely low birth weight infants. Fetal Pediatr Pathol 2016;35:299-306.

13. Laughon SK, Reddy UM, Sun L, Zhang J. Precursors for late preterm birth in singleton gestations. Obstet Gynecol 2010;116:1047-55. 